\title{
Comparative study between measurement and predictions using geometrical optics and uniform theory of diffraction for case of non-line-of-sight (NLOS) in indoor environment
}

\begin{abstract}
This paper describes the investigation and comparison of the accuracy of a deterministic model for a WLAN system in the indoor environment. The measurement system consisted of a spectrum analyzer and a log-periodic antenna. Non-line-of-sight (NLOS) propagation (in furnished rooms) was investigated. All the measurement sites mentioned in this paper are located in the Division of Information Technology at Universiti Putra Malaysia. The furnished rooms mentioned above were a teaching laboratory and a computer laboratory. The measured path losses were compared with the results obtained using deterministic models, namely the geometrical optics model and the uniform theory of diffraction model with the aid of image theory. The predicted results showed good agreement with the measured data for the NLOS environment, with an absolute mean error that ranged between 1.61 and $3.07 \mathrm{~dB}$.
\end{abstract}

Keyword: Diffraction; Geometrical optics; Uniform theory of diffraction 